\title{
NovelAgents for the Treatment of Hereditary Hemorrhagic Telengiectasia: Are They Effective Enough?
}

Burak Uz*

*Gazi University Faculty of Medicine, Department of Internal Medicine, Division of Adult Hematology

${ }^{\star}$ Correspondence to: Burak Uz, Emniyet Mah, Gazi University Faculty of Medicine, Department of Internal Medicine, Division of Adult Hematology, Turkey; Tel: +90 (312) 20255 79; +90 (312) 20263 17; Fax: +90 (312) 22367 14; E-mail: burakuz78@gmail.com

Received: August 13, 2016; Accepted: September 17, 2016; Published: October 02, 2016

\section{Editorial}

Hereditary hemorrhagic telangiectasia (HHT) is a relatively rare $(1 / 5000)$ autosomal dominant disorder characterised with arteriovenous malformations located in mucosal areas, gastrointestinal tract, pulmonary, hepatic and cerabral circulations. Several mutations (endoglin, activin A receptor type II-like 1, and less frequently SMAD4 gene) are determined, however the main cause of HHT remains unclear $[1,2]$. Current guideliness recommend to take measures for the prevention of bleeding, avoidance of antiplatelet or anticoagulant agents (if possible), electrical or chemical coagulation techniques via endoscopic procedures, and treatment of iron-deficiency anemia [2].

Circulating vascular endothelial growth factor (VEGF) levels are elevated in HHT. Recently bevacizumab, a VEGF inhibitor, was administered parenterally to patients with HHT in order to investigate whether it has systemic anti-angiogenic effects in HHT. The bevacizumab dose administered was the same as its usual dose recommended for metastatic colorectal cancer patients $(5 \mathrm{mg} / \mathrm{kg}$, every 2 weeks, for 6 cycles). Among the evaluable subjects $(n=5)$, all patients had a decrease in transfusion requirements, and one of them gained transfusion independency. Improvement in hemoglobin levels were detected 2 months following bevacizumab administration. Some patients $(n=2)$ needed additional infusions of bevacizumab, but no serious (grade III or IV) side effects were reported. Bevacizumab was found to be more effective in patients suffering from epistaxis [3]. To avoid its systemic side-effects bevacizumab was also administered locally. In ELLIPSE phase-1 study which was performed on 40 HHT patients, nazal bevacizumab was well tolerated but showed no efficacy at the doses of $12.5,25,50,75$, and $100 \mathrm{mg} / \mathrm{mL}$, respectively [4]. Subsequently, in a placebo-controlled and double-blind study with 15 HHT patients, a single intranasal submucosal bevacizumab injection $(10 \mathrm{~mL}, 100 \mathrm{mg})$ reduced epistaxis severity and visual analog scores (27\% vs $3 \%)$ non-significantly [5].

Beyond its immunomodulatory effects, thalidomide has antiangiogenenic potency, and also suppresses tumor necrosis factor alpha [1]. The efficacy of thalidomide in preventing gastrointestinal hemorrhages from angiodysplasias was proven in 2011. This prospective study included 2 arms, $100 \mathrm{mg}$ /day thalidomide versus 400 $\mathrm{mg} /$ day iron (controls) were administered for 4 months. As expextedly, the subjects in thalidomide arm had significantly higher response rate which was described as a decrease by $\geq 50 \%$ in bleeding episodes.
Thalidomide treatment was associated with a reduction in VEGF levels without any serious adverse events [6]. These promising results led clinicians to administer thalidomide for patients with HHT. Although no randomised clinical trial is reported, some small case series have emerged its clinical efficacy in HHT patients presenting with epistaxis at a dose of 50-250 mg/day (most commonly 100-200 mg/day). These patients have also showed less transfusion requirements and have described improvements in quality of life scores [7]. Special attention needs be paid while prescribing thalidomide. Teratogenicity, sedation, peripheral sensorial neuropathy, hematologic cytopenias, and venous thrombosis are the potential adverse effects of thalidomide. $100 \mathrm{mg} /$ day thalidomide should be used to avoid from neuropathy instead of $300 \mathrm{mg} /$ day. Interestingly, thromboembolic events were not reported in previous studies including patients with HHT [1].

Lenalidomide, a novel immunomodulatory drug which has less side effects than thalidomide, was successfully used in a 69-year-old women with chronic gastrointestinal bleeding due to HHT. Initially she was under thalidomide therapy (50-100 mg/day), but the drug was stopped because of thalidomide-induced grade- 3 peripheral neuropathy. She has became free of gastrointestinal hemorrhage and drug side effects, with higher hemoglobin values (10.9 vs. 13.2 $\mathrm{g} / \mathrm{dL}$ ) and reduced iron and blood transfusion requirements after a 13-months period of lenalidomide treatment [8]. Both thalidomide and lenalidomide carry anti-angiogenic properties in a dose-dependent manner [9]. Additionally, the inhibitory potency of lenalidomide on growth factor-induced Akt phosphorylation and anti-migratory effects on endothelial cells were clearly shown in a rat mesenteric window assay by Dredge and colleagues [10]. Lenalidomide can suppress bone marrow activity, and therefore individual dosing should be monitored according to regular blood counts. However, high treatment costs, lack of endication in patients with HHT regarding health insurance, and most importantly limited evidence-based data about the efficacy of lenalidomide in HHT should be kept in mind.

In conclusion, novel agents such as bevacizumab or IMIDs should be used cautiously because of their potential side effects. Bevacizumab and thalidomide seems to be effective in HHT patients presenting with epistaxis. However, IMIDs could be preferred in gastrointestinal hemorrhages in HHT. These new treatment options will also provide improvements in quality of life in HHT. Although serious side effects are not reported in the recommended doses of these agents, there is 
insufficient evidence to recommend them as first line therapy. Further randomised and well-designed studies are needed to better identify the optimal treatment modality for the prevention and treatment of bleeding episodes in HHT.

\section{References}

1. Bauditz J (2016) Effective treatment of gastrointestinal bleeding with thalidomide-Chances and limitations. World J Gastroenterol 22: 3158-3164. [crossref]

2. Faughnan ME, Palda VA, Garcia-Tsao G, Geisthoff UW, McDonald J, et al. (2011) International guidelines for the diagnosis and management of hereditary haemorrhagic telangiectasia. J Med Genet 48: 73-87. [crossref]

3. Epperla N, Kapke JT, Karafin M, Friedman KD, Foy P (2016) Effect of systemic bevacizumab in severe hereditary hemorrhagic telangiectasia associated with bleeding. Am J Hematol 91:E313-4.

4. Dupuis-Girod S, Ambrun A, Decullier E, Samson G, Roux A, et al. (2014) ELLIPSE Study: a Phase 1 study evaluating the tolerance of bevacizumab nasal spray in the treatment of epistaxis in hereditary hemorrhagic telangiectasia. MAbs 6:794-9.

5. Riss D, Burian M, Wolf A, Kranebitter V, Kaider A, et al. (2015) Intranasal submucosal bevacizumab for epistaxis in hereditary hemorrhagic telangiectasia: a double-blind, randomized, placebo-controlled trial. Head Neck 37:783-7.

6. Ge ZZ, Chen HM, Gao YJ, Liu WZ, Xu CH, et al. (2011) Efficacy of thalidomide for refractory gastrointestinal bleeding from vascular malformation. Gastroenterology 141:1629-37.e1-4.

7. Franchini M, Frattini F, Crestani S, Bonfanti C (2013) Novel treatments for epistaxis in hereditary hemorrhagic telangiectasia: a systematic review of the clinical experience with thalidomide. J Thromb Thrombolysis 36:355-7.

8. Bowcock SJ, Patrick HE (2009) Lenalidomide to control gastrointestinal bleeding in hereditary haemorrhagic telangiectasia: potential implications for angiodysplasias? Br J Haematol 146:220-2.

9. Dredge K, Marriott JB, Macdonald CD, Man HW, Chen R,et al. (2002) Novel thalidomide analogues display anti-angiogenic activity independently of immunomodulatory effects. Br J Cancer 87:1166-72.

10. Dredge K, Horsfall R, Robinson SP, Zhang LH, Lu L, et al. (2005) Orally administered lenalidomide (CC-5013) is anti-angiogenic in vivo and inhibits endothelial cell migration and Akt phosphorylation in vitro. Microvasc Res 69:56-63. 